\title{
A NEW APPROACH FOR CALCULATING THE ACCESSIBLE VOLUME IN EQUATIONS OF STATE FOR MIXTURES. I. THEORY AND IMPLEMENTATION IN THE VAN DER WAALS EQUATION OF STATE
}

\author{
B. SMIT and K.R. COX $\star$ \\ Koninklijke/Shell-Laboratorium, Amsterdam Shell Research B.V., P.O. Box 3003, \\ 1003 AA Amsterdam (The Netherlands)
}

(Received November 10, 1987; accepted in final form March 18, 1988)

\begin{abstract}
Smit, B. and Cox, K.R., 1988. A new approach for calculating the accessible volume in equations of state for mixtures. I. Theory and implementation in the van der Waals equation of state. Fluid Phase Equilibria, 43: 171-180.

In this paper we present a generalization of the van der Waals equation of state for mixtures based on a new concept of calculating the accessible volume of the molecules. The conventional approach for calculating the accessible volume is based on hard-spheres models. With such models it is impossible to incorporate directly the influence of binary interactions which affect the volume occupied by the molecules. On the basis of the original ideas of van der Waals we introduce the concept of apparent size as probed by a particular molecule, in order to approximate the excluded volume for mixtures. With this new concept it is possible to describe the influence of binary interactions on the accessible volume directly.
\end{abstract}

\section{INTRODUCTION}

It was more than a century ago that van der Waals proposed his famous equation of state (van der Waals, 1873) to explain the continuity of the liquid and the vapour phase. The elegance and physical insight with which he was able to correct the ideal gas law for the repulsion and attraction of the molecules have contributed to the fact that the basic form of his equation is still used in many other, generally more empirical, equations of state. This fact becomes even more amazing if we realize that in the original form the equation was only used to describe the behaviour of simple fluids

\footnotetext{
‡ Present address: Shell Development Company, Westhollow Research Center, P.O. Box 1380, Houston, TX 7751-1380, U.S.A.
}

$0378-3812 / 88 / \$ 03.50$

(C) 1988 Elsevier Science Publishers B.V. 
(noble gases). Nowadays, modifications of the van der Waals equation are used to describe the behaviour of mixtures of very complicated molecules (Prausnitz, 1969).

A great deal of work has been directed towards extending the van der Waals equation and related equations to include mixtures. It is generally accepted that the same equation can be used for the description of mixtures. The specific interactions of the molecules must be included in appropriate mixing rules for the parameters of the equation of state. The development of these mixing rules has received considerable attention over the last few years (Mansoori, 1986).

In the development of these mixing rules it is normally assumed that the mixing rule for the $b$ parameter can be estimated from the pure components with a hard-spheres model. Specific binary interactions between the molecules are considered in the mixing rule of the $a$ parameter (Mansoori and Ely, 1985). In practical applications these interactions are incorporated in a so-called binary interaction coefficient. Some authors (Deiters and Schneider, 1976; de Loos et al., 1984; Peters, 1986) have shown that for mixtures of molecules which differ substantially in size and shape, a second binary interaction coefficient is necessary to describe these systems accurately. This second coefficient is introduced in the mixing rule for the $b$ parameter in order to correct the accessible volume for specific binary interactions.

In this paper we will discuss the behaviour of a mixture in which binary interactions affect the "effective size" of the molecules. This behaviour is modelled with a Lennard-Jones mixture, by introducing a coupling parameter in the potential which describes the unlike interactions.

The limiting behaviour of this mixture cannot be described properly with an equation of state due to a hard-spheres model in the expression for the $b$ parameter. In this paper we demonstrate that a generalization of the equation of state, which is based on the ideas of two-fluid theory, can resolve this. Furthermore, this generalization allows us to introduce a binary interaction coefficient in the expression for the $b$ parameter. This coefficient has a direct physical interpretation and can be related to the intermolecular potential, unlike the currently-used interaction coefficient (Peters, 1986).

We have, for simplicity, chosen the van der Waals equation to illustrate this behaviour. Although using this equation implies several simplifications, we want to emphasize that our results are not limited to this equation. The same arguments can be applied to all equations of state which are based on the principles of the van der Waals equation and which use hard sphere mixing rules.

The remainder of this paper is organized in the following way. First a brief review is given of the relation of the van der Waals mixing rules and the intermolecular potential. Next we demonstrate that the ordinary mixing 
rules with the van der Waals equation of state cannot properly describe a hypothetical mixture in which binary interactions affect the "effective size" of the molecules. Finally, we propose a generalization which resolves this inconsistency.

THE VAN DER WAALS EQUATION OF STATE FOR MIXTURES

In most practical applications, fluid phase behaviour of mixtures is calculated by means of equations of state which are modifications of the original van der Waals equation

$p=R T /(v-b)-a / v^{2}$

where $a$ and $b$ are the van der Waals parameters of a pure fluid. This equation can also be used for mixtures if we assume that a mixture behaves like a hypothetical pure fluid (conformal solution theory). The van der Waals parameters of this hypothetical fluid are obtained from the familiar mixing rules

$a=\sum_{i} \sum_{j} x_{i} x_{j} a_{i j}$

$b=\sum_{i} \sum_{j} x_{i} x_{j} b_{i j}$

where $x_{i}$ is the mole fraction of compound $i$ and the parameters for the unlike $i-j$ interactions are given by

$a_{i j}=\left(a_{i i} a_{j j}\right)^{1 / 2}$

$b_{i j}=\left(b_{i i}+b_{j j}\right) / 2$

It is well known that the principles of corresponding states are not limited to a macroscopic equation of state. These principles can be extended to a microscopic theory of corresponding states, which is based on the mathematical properties of the intermolecular potential.

Assume that from the intermolecular potential a hard-spheres contribution can be separated

$U_{i j}=U^{\mathrm{HS}}\left(\sigma_{i j}\right)+\epsilon_{i j} F\left(r_{i j} / \sigma_{i j}\right)$

Equating the radial distribution functions of the $i, j$ pairs to that of a pure fluid and expanding the Helmholtz free energy of the mixture and the 
reference fluid about that of a hard-spheres fluid leads to the van der Waals mixing rules

$\epsilon_{\mathrm{m}} \sigma_{\mathrm{m}}^{3}=\sum_{i} \sum_{j} x_{i} x_{j} \epsilon_{i j} \sigma_{i j}^{3}$

$\sigma_{\mathrm{m}}^{3}=\sum_{i} \sum_{j} x_{i} x_{j} \sigma_{i j}^{3}$

with

$\epsilon_{i j}=\left(\epsilon_{i i} \epsilon_{j j}\right)^{1 / 2}$

$\sigma_{i j}=\left(\sigma_{i i}+\sigma_{j j}\right) / 2$

It can be shown (Mansoori, 1986) that mixing rules (2), (3), (4) and (5) are related to the equations above ( $a \propto \epsilon \sigma^{3}$ and $b \propto \sigma^{3}$ ).

HYPOTHETICAL LENNARD-JONES MIXTURE,

The Lennard-Jones (6-12) potential is often used to model the behaviour of non-polar simple liquids

$U_{i j}(r)=4 \epsilon_{i j}\left[\left(\sigma_{i j} / r\right)^{12}-\left(\sigma_{i j} / r\right)^{6}\right]$

where $\epsilon_{i j}$ is the characteristic energy parameter for the $i-j$ interactions and $\sigma_{i j}$ is the characteristic distance parameter for the $i-j$ interactions.

For estimating the characteristic parameters for the unlike interactions the Lorentz-Berthelot combining rules (eqns. (9) and (10)) are normally used.

In this paper we are interested in the behaviour of a mixture in which binary interactions affects the "effective size" of the molecules. We have modelled this behaviour by introducing a coupling parameter $\lambda_{i j}$ (Shing and Gubbins, 1982) in the combining rule in the Lennard-Jones potential for the unlike interactions

$\epsilon_{i j}=\lambda_{i j}\left(\epsilon_{i i} \epsilon_{i j}\right)^{1 / 2}$

In Fig. 1 the Lennard-Jones potential is plotted for several values of the coupling parameter. Note that varying $\lambda$ not only affects the depth of the well of the potential but also shifts the soft-core diameter.

In order to simplify the system under consideration, we will restrict our discussion to a binary system, in which both pure components are assumed to have the same characteristic size and energy parameter for all values of $\lambda_{i j}$

$\sigma_{12}=\sigma_{11}=\sigma_{22}$ and $\epsilon_{11}=\epsilon_{22}$ 


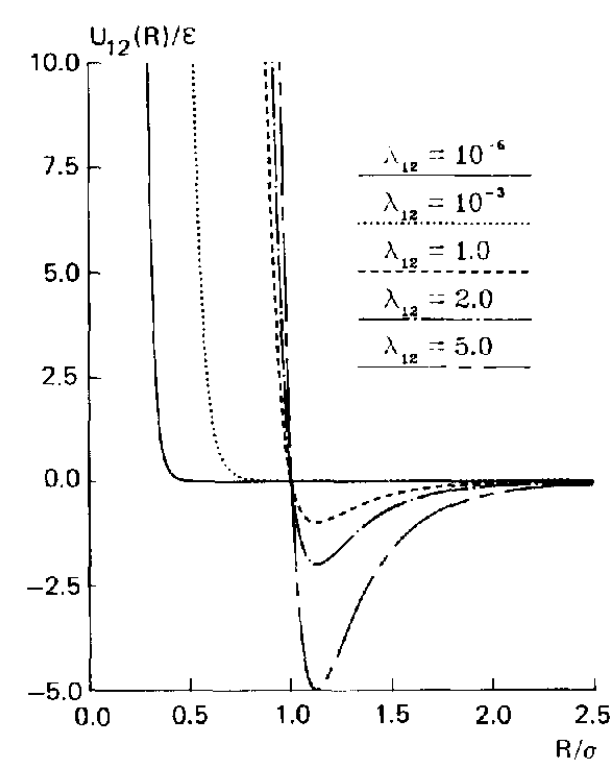

Fig. 1. The Lennard-Jones potential for several values of the coupling parameter $\lambda_{12}$.

Extension to a multicomponent system does not give rise to additional complications.

For this system we can distinguish two limiting cases. In the limit of $\lambda_{12}=1$ there is no difference in the $1-1,2-2$ and $1-2$ interactions. The system behaves like a pure Lennard-Jones fluid. For the mixing rule of the van der Waals equation (eqns. (2) and (3)) this implies

$a=a_{11}=a_{22}=a_{12}$ and $b=b_{22}=b_{11}=b_{12}$

and, obviously, the equation of state for the mixture becomes identical with the van de Waals equation for pure fluids.

In the second limit, $\lambda_{12}=0$, there are no 1-2 interactions. Particles 1 behave completely independently of particles 2 . Note that in this limit the system does not reduce to a mixture of ideal gases, but to a mixture of pure components which do not interact with each other. So we expect the expression for the total pressure to be just the sum of the pressures of pure component 1 and pure component 2.

The combining rule of van der Waals' $a$ parameter for this case $\left(\lambda_{12}=0\right)$ reads

$a_{21}=0$

With this combining rule, the van der Waals equation reduces to

$$
\begin{aligned}
p / R T= & x_{1} /\left(v-x_{1} b_{1}-x_{2} b_{2}\right)-x_{1}^{2} a_{11} / R T v^{2} \\
& +x_{2} /\left(v-x_{1} b_{1}-x_{2} b_{2}\right)-x_{2}^{2} a_{22} / R T v^{2}
\end{aligned}
$$


This equation states a result which is inconsistent with the physical picture of the $\lambda_{12}=0$ limit. In this limit, the two components are non-interacting and should make independent contributions to the presssure. So it should be possible to write

$p=x_{1} p_{1}+x_{2} p_{2}$

with no parameters for component 2 appearing in the expression for $p_{1}$ and vice versa. Yet, in the first term on the right-hand side of eqn. (14) the accessible volume of the 1-particles is given as the total volume of the system $(v)$ minus the sum of the "hard core" excluded volume of the 1-particles $\left(x_{1} b_{1}\right)$ and the "hard core" excluded volume of the 2-particles $\left(x_{2} b_{2}\right)$. Although the particles are by definition non-interacting, this equation predicts an accessible volume which depends on the other species for the limit $\lambda_{12}=0$.

The reason for the incorrect behaviour in this limit becomes clear if we realize that in the derivation of the van der Waals mixing rules it is assumed that the radial distribution functions can be expanded around that of a hard sphere. For the unlike interactions this leads to a hard sphere diameter given by eqn. (10). However, in the limit $\lambda_{12}=0$ inspection of the intermolecular potential shows that the effective hard sphere radius approaches zero, independent of $\sigma_{11}$ and $\sigma_{22}$. Therefore, the hard-spheres mixing rules (eqns. (3) and (5)) for the $b$ parameter cannot give good estimates for this limit.

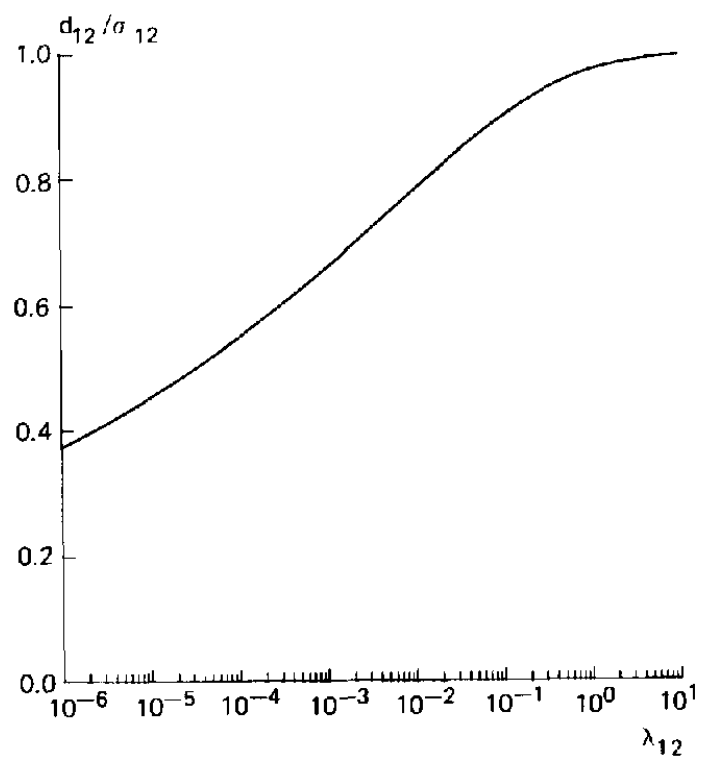

Fig. 2. The effective hard-sphere diameter $d_{12}$ as a function of the coupling parameter $\lambda_{12}$. 
For a given potential Barker and Henderson (1967) define an effective hard-sphere diameter $d_{i j}$ as

$d_{i j}=\int_{0}^{\sigma}\left\{1-\exp \left[-\beta U_{i j}(r)\right]\right\} \mathrm{d} r$

where $\sigma$ is the value of $r$ for which $U_{i j}(r)$ is zero and $\beta$ is $1 / k T$.

In Fig. 2 the apparent size of the particles is given as a function of $\lambda_{12}$. Note that if we consider a particle 1 the apparent size of this particle is independent of $\lambda_{12}$ when probed by another particle 1 , but its apparent size is dependent on $\lambda_{12}$ when probed by a particle 2 . This figure demonstrates that for this system a hard-spheres model based on the pure fluids cannot give correct estimates of the excluded volume.

GENERALIZATION OF THE VAN DER WAALS EQUATION OF STATE FOR MIXTURES

In the previous section we have demonstrated that the van der Waals equation cannot describe qualitatively a hypothetical Lennard-Jones mixture, because of the hard-spheres approximation in the mixing rule for the $b$ parameter. In this section we will present a generalization of the van der Waals equation which can resolve this inconsistency.

In the original derivation of the van der Waals equation for pure compounds the $b$ parameter is introduced to exclude the volume occupied by the molecules. The accessible volume for a molecule is the total volume minus the volume excluded by all other molecules. For a mixture of hard spheres, the accessible volume for all species can be estimated from the pure components.

In the Lennard-Jones system we are considering here the concept of apparent size is introduced to express the influence of the unlike interaction on the effective size of the molecules. With this concept of size, it is more logical to state that the accessible volume for a molecule $i$ is the total volume minus the sum of the total apparent volume of $i$-particles as probed by particle $i$ (independent of $\lambda$ ) and the total apparent volume of the $j$-particles as probed by this particle $i$ (dependent on $\lambda$ ) and vice versa for the apparent volume of the molecules $j$. Note that this approach for calculating the accessible volume is related to the idea of considering two reference state as is used in two-fluid theory for calculating the extensive configurational properties (Scott, 1956; Leland et al., 1968, 1969)

Implementation of this concept in the van der Waals equation for a multicomponent system gives

$$
\begin{aligned}
& p / R T=\sum_{i} x_{i} p_{i} / R T \\
& p_{i} / R T=1 /\left(v-\sum_{j} x_{j} b_{i j}\right)-\sum_{j} x_{j} a_{i j} / R T v^{2}
\end{aligned}
$$


For a binary mixture this equation reads

$$
\begin{aligned}
p / R T= & x_{1} /\left(v-x_{1} b_{11}-x_{2} b_{12}\right)-x_{1}^{2} a_{11} / R T v^{2}-2 x_{1} x_{2} a_{12} / R T v^{2} \\
& +x_{2} /\left(v-x_{1} b_{21}-x_{2} b_{22}\right)-x_{2}^{2} a_{22} / R T v^{2}
\end{aligned}
$$

where $b_{12}$ indicates the apparent excluded volume of a 2-particle as probed by a 1-particle, and $b_{21}$ indicates the apparent excluded volume of a 1-particle as probed by a 2-particle.

The apparent volume of the particles can be estimated from the intermolecular potential by eqn. (15). In this expression for the effective hard sphere diameter the exchange of 1 and 2 has no influence, so $b_{12}=b_{21}$

If we now consider the limit $\lambda_{12}=0$, inspection of Fig. 2 and the intermolecular potential shows:

$a_{12}=0$ and $b_{12}=b_{21}=0$

If these values are substituted in eqn. (17), we find

$p / R T=x_{1} /\left(v-x_{1} b_{11}\right)-x_{1}^{2} a_{11} / R T v^{2}+x_{2} /\left(v-x_{2} b_{22}\right)-x_{2}^{2} a_{22} / R T v^{2}$

and we see that the total pressure is the sum of the (independent) contributions to the pressure of the 1-particles and 2-particles.

Evidently, implementation of the concept of apparent volumes of the molecules in the van der Waals equation demonstrates a correct qualitative behaviour in the limiting case that $\lambda=0$. But the concept of apparent size also relaxes the assumption of a hard-spheres model in the derivation of the mixing rule for the $b$ parameter of an equation of state. Accordingly, it is interesting to see to what extent this concept can be generalized to more realistic molecules.

It is interesting to note that the generalized van der Waals equation (eqn. (16)) becomes identical with the normal van der Waals equation with the ordinary mixing rule for the $b$ parameters $(1, \ldots, 5)$ if it is assumed that the apparent volume of particle $i$ is independent of the particle by which it is probed (hard-spheres approximation). This implies for the $b_{i j}$ parameters of the generalized equation that

$b_{j i}=b_{i i}$ for every value of $j$

Substitution of these parameters into eqn. (16) gives the ordinary van der Waals equation.

\section{DISCUSSION AND CONCLUSIONS}

In this report we have presented a new approach for calculating the accessible volume, in which the concept of apparent size as probed by a 
particular molecule is introduced. Implementation of this concept in the van der Waals equation leads to a generalization which relaxes the hard-spheres approximation in the mixing rule of the $b$ parameter. With this new equation it is in principle possible to describe the influence of binary interactions on the accessible volume of the molecules. In a subsequent paper we will present an application of this approach to illustrate this.

We speculate that many systems which are known for their peculiar specific binary interactions can be better described with an equation of state by the introduction of this new concept. One may think of equations, such as the well-known Peng-Robinson or Soave-Redlich-Kwong, which are often used in practical applications.

It is worthwhile to note that a binary interaction parameter $\left(l_{i j}\right)$ can be introduced in the expression for $b_{i j}$ (eqn. (16)):

$b_{i j}=\left(1-l_{i j}\right) b_{j j}$

This interaction parameter can be regarded as a correction to the hardspheres value $b_{i i}$ and can be related to the intermolecular potential by means of this new concept of apparent size. This is in contrast to the previously introduced (Deiters and Schneider, 1976; Peters, 1986) interaction parameter which was a purely empirical-fit parameter.

\section{LIST OF SYMBOLS}

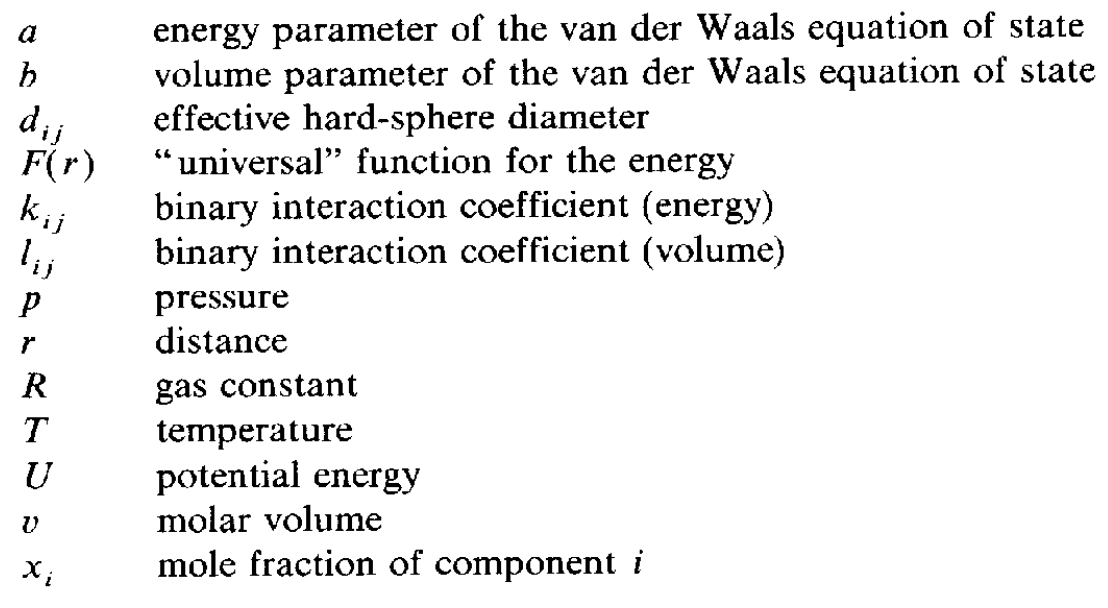

Greek letters

$\beta \quad$ reciprocal temperature

$\epsilon_{i j} \quad$ characteristic energy parameter for the $i-j$ interactions 
$\xi_{i j} \quad$ binary interaction coefficient (combining rule of the characteristic energy)

$\lambda_{i j} \quad$ coupling parameter

$\sigma_{i j} \quad$ characteristic distance parameter for the $i-j$ interactions

\section{ACKNOWLEDGEMENTS}

The authors gratefully acknowledge the contributions of Dr. J.P.J. Michels from the van der Waals Laboratorium of the University of Amsterdam, and Drs. J.F. Ely and V. van de Leeuw for constructive criticism of an earlier version of this manuscript.

\section{REFERENCES}

Barker, J.A. and Henderson, D., 1967. Perturbation theory and equations of state for fluids. II. A successful theory of liquids. J. Chem. Phys., 47: 4714-4721.

Deiters, U. and Schneider, G.M., 1976. Fluid mixtures at high pressures. Computer calculations of the phase equilibria and the critical phenomena in fluid binary mixtures from the Redlich-Kwong equation of state. Ber. Bunsenges. Ges., 80: 1316-1321.

De Loos, Th.W., Poot, W. and Lichtenthaler, R.W., 1984. Fluid phase equilibrium in binary ethylene + n-alkane systems. Ber. Bunsenges. Phys. Chem., 88: 855-859.

Leland, T.W., Rowlinson, J.S. and Sather, G.A., 1968. Statistical thermodynamics of mixtures of molecules of different sizes. Trans. Faraday Soc., 64: 1447-1460.

Leland, T.W., Rowlinson, J.S., Sather, G.A. and Watson, I.D., 1969. Statistical thermodynamics of mixtures of two-fluid models mixtures. Trans. Faraday Soc., 65: 2034-2043.

Mansoori, G.A., 1986. Mixing rules for cubic equations of state. In: K.C. Chao and R.L. Robinson Jr. (Editors), Equations of State: Theory and Applications. ACS Symp. Ser. No. 300, Am. Chem. Soc., Washington, pp. 314-330.

Mansoori, G.A. and Ely, J.F., 1985. Density expansion (DEX) mixing rules: thermodynamic modeling of supercritical extraction. J. Chem. Phys., 82: 406-413.

Peters, C.J., 1986. Phase behaviour of binary mixtures of ethane + n-eicosane and statistical mechanical treatment of Muid phases. Thesis, University of Delft.

Prausnitz, J.M., 1969. Molecular Thermodynamics of Fluid-Phase Equilibria. Prentice-Hall, Englewood Cliffs.

Scott, R.L., 1956. Corresponding states treatment of nonelectrolyte solutions. J. Chem. Phys., 25: $193-205$.

Shing, K.S. and Gubbins, K.E., 1982. The chemical potential in dense fluids and fluid mixtures via computer simulations. Mol. Phys., 96: 1109-1128.

Van der Waals, J.D., 1873. Over de continuiteit van den gas-en vloeistoftoestand. Thesis, University of Leiden. 